\title{
Neutral density estimation in the ASDEX Upgrade divertor from deuterium emissivity measurements
}

\author{
M.Agostini ${ }^{1}$, N.Vianello ${ }^{1}$, L.Carraro ${ }^{1}$, D.Carralero ${ }^{2}$, M.Cavedon $^{3}$, R.Dux ${ }^{3}$, V.Naulin ${ }^{4}$, \\ M.Spolaore ${ }^{1}$, E.Wolfrum ${ }^{3}$, the ASDEX Upgrade Team, the EUROfusion MST1 Team ${ }^{5}$ \\ ${ }^{1}$ Consorzio RFX, C.so Stati Uniti 4, 35127, Padova, Italy \\ ${ }^{2}$ CIEMAT Laboratorio National de Fusion, \\ Avda. Complutense 40, 28040 Madrid, Spain \\ ${ }^{3}$ Max-Planck-Institut für Plasmaphysik, 8574, Garching, Germany \\ ${ }^{4}$ Association Euratom DTU, Technical University of Denmark, Roskilde, Denmark and \\ ${ }^{5}$ See the author list H. Meyer et al 2017 Nucl. Fusion 57102014
}

(Dated: April 12, 2019)

\begin{abstract}
Neutral density distribution in the divertor region of ASDEX Upgrade is estimated in different discharge conditions. The 2D divertor emission of $D_{\alpha}$ and $D_{\gamma}$ is obtained with a tomographic inversion of their brightness measured by two cameras. From these emissions, 2D maps of neutrals density $n_{0}$ can be obtained by a simple model that takes into account both excitation and recombination processes populating deuterium $n=3$ and $n=5$ levels. In attached discharges, the neutral density $n_{0}$ is larger in the inner divertor, and when increasing core $n_{e}$ its maximum moves toward the dome and the outer divertor region. In particular, the modification of the $n_{0}$ distribution is studied in plasmas where the mean electron density in the core increases, moving from an attached to a detached phase. In these discharges, the outboard midplane scrape off layer electron density increases and its profile flattens, with the formation of the so called density shoulder. The modification of the neutral density is followed during this evolution, showing that both the shape and the absolute value of the $n_{0}$ distribution change in time, suggesting that they play a role in the two phenomena of shoulder formation and detachment. Moreover, in the detached phase, also the recombination contributes to the neutral deuterium emission.
\end{abstract}




\section{INTRODUCTION}

In current tokamak experiments and in future devices the control of the plasma-wall processes and plasma-wall interaction (PWI) are a key issue. In the divertor region, where PWI is strongest, energy and particle fluxes have to be reduced up to value manageable by materials, without damages and breaking [1]. A promising solution consists in high density operation, which is associated to a regime where the plasma detaches locally from the divertor, causing a strong reduction of power fluxes into the divertor targets [2-5]. In this scenario, the interaction between divertor plasma and neutrals seems to play an important role $[6,7]$. In fact interaction with neutrals could explain the so-called shoulder formation: a broadening and flattening of the electron density profile in the Scrape-Off Layer (SOL) at the outboard midplane, usually observed at high density operations and associated with detachment $[8,9]$. As described in [7], the shoulder formation can be due to neutral-plasma interaction. With the increase of the divertor $n_{0}$, also the ionisation increases, leading to lower flows into the divertor; this mechanism can reduce the loss of ions from the upstream SOL, with the consequent increase of upstream SOL $n_{e}$. Another possible mechanism is that when the divertor $n_{0}$ increases, the mean free path for ionization and the parallel collision length become shorter, and the probability for a neutral to be ionized near the separatrix and fueling the core decreases. In order to maintain the same core density, the inward neutral flux must increase; this leads to more ionization in the SOL, with the consequent increase in density and decrease in the mean free path $[10,11]$.

Considering the importance of divertor neutrals, the paper describes a method to infer the neutral density $n_{0}$ from the emissivity of $D_{\alpha}$ and $D_{\gamma}$ measured by cameras in the ASDEX Upgrade tokamak. Plasma discharges with shoulder formation and detachment obtained by deuterium puffing and electron density increase are studied. The use of Balmer lines for obtaining information about neutrals, ionization and recombination in the tokamak edge and divertor is well documented, and it is widely used for studying divertor detachment (without being a complete bibliography, see for example [12-18]).

In the next section the visible cameras are described, together with the tomographic algorithm developed for obtaining 2D emissivity profile from line integrated measurements; section III describes the technique for the estimation of $n_{0}$; the results obtained in discharges with density ramps are shown in section IV. The final discussion is contained in sec. V. 


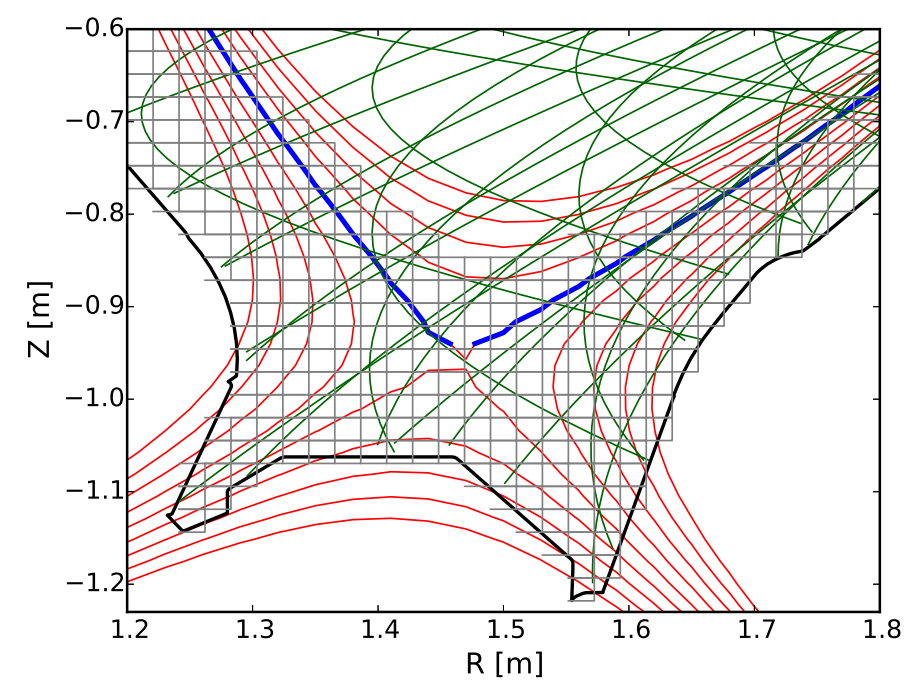

Figure 1: Geometry used for tomographic algorithm in the divertor region. The vessel is shown in black; the green lines are the projections in the $(R, Z)$ plane of a selection of lines of sight of the $D_{\alpha}$ camera positioned in the outboard equatorial midplane. Flux surfaces are shown in red, together with the separatrix (thicker line). The gray lines reproduce the pixels division and so the spatial resolution of the tomography.

\section{TOMOGRAPHIC INVERSION}

ASDEX Upgrade is equipped with many cameras looking at different regions and at different wavelengths. In particular there are two visible cameras with two filters for $D_{\alpha}$ and $D_{\gamma}$ lines. They observe the divertor region from the outboard midplane, and they are absolute calibrated. Since they observe along lines of sight (LoSs), they measure integrated signals, and in order to obtain a 2D emission map $\epsilon$, a tomographic algorithm has been developed. Assuming that the emission does not depend on the toroidal angle $\phi$, we have $\epsilon=\epsilon(R, Z)$; in this way it is possible to project each LoS corresponding to each camera pixel in the plane $(R, Z)[19]$, as shown in figure 1 . In this figure some of the LoSs in the divertor region are shown in green, together with the flux surfaces as an example. As highlighted in the figure, in the $(R, Z)$ plane the LoSs are not straight and they intersect each other: thus this is a good starting point for developing a tomographic algorithm.

Within the different possibilities in the choice of the algorithm [20-23], the pixel method has been preferred, in order to introduce as little as possible a-priori constraints, together with an algebraic iterative technique. In the development of the inversion algorithm, two assumptions are made: the $D_{\alpha}$ and $D_{\gamma}$ emissions do not depend on the toroidal angle and they are restricted outside the separatrix. Thus the divertor region outside the separatrix is divided into rectangular pixels (see 

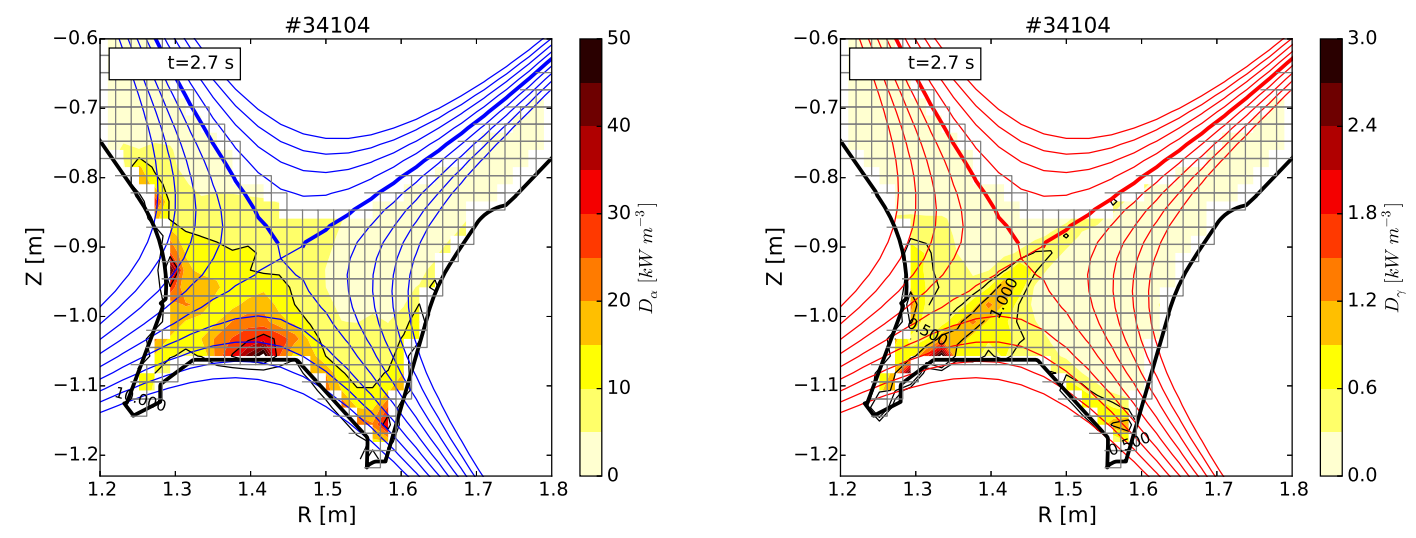

Figure 2: Example of $2 \mathrm{D} \epsilon_{\alpha}$ (left) and $\epsilon_{\gamma}$ (right) profiles obtained via tomographic inversion for shot \#34104 at $t=2.7 \mathrm{~s}$. Red and blue lines are the flux surfaces with the separatrix (thicker one).

figure 1) and the emissivity $\epsilon$ in each pixel is considered constant. It is important to highlight that no other information about the magnetic equilibrium is added.

Using this method, the link between the intensity measured by each LoS of the camera and the emissivity is a linear system of equations, that can be written as:

$$
\mathbf{I}=\mathbf{A} \cdot \epsilon
$$

where $I_{j}$ is the line integrated signal measured by the LoS $j ; \epsilon_{i}$ is the unknown emissivity of the pixel $i$; and the matrix element $a_{i j}$ is the length of the $\operatorname{LoS} j$ inside the pixel $i$. This matrix is evaluated only once, since it depends on the geometry of the LoSs and the pixels division. In eq. 1 there are $\approx 40000$ LoSs and $\approx 200$ pixels, so the system is over-determined. For inverting it and obtaining the emissivity of each pixel, the simultaneous algebraic reconstruction technique (SART) is used $[24,25]$. It is an iterative technique which allows solving the linear system of Eq. 1 via an iterative error-correcting procedure, according the formula:

$$
\epsilon_{i}^{k+1}=\epsilon_{i}^{k}+\frac{\Sigma_{j}\left[a_{i j} \frac{\mathbf{I}_{j}-\mathbf{a}_{j} \cdot \epsilon^{(k)}}{\Sigma a_{i j}}\right]}{\Sigma_{j} a_{i j}}
$$

where $\epsilon_{i}^{k}$ is the emissivity of the pixel $i$ after $k$ iterations. The initial estimate $\epsilon_{i}^{0}$ is set to 0 . Since the expected emissivity of each pixel is positive, this constraint is enforced by setting to zero the coefficients that are lower than zero after each iteration step. The convergence is quite rapid, and it is reached after about 20 iterations. Moreover, since the number of LoSs is much more larger than the unknowns, regularization is not needed; this can be seen by the inverted images which do not present any large difference between adjacent pixel.

An example of the emissivities $\epsilon_{\alpha}$ and $\epsilon_{\gamma}$ obtained in the divertor with the inversion is shown in 
figure 2: left panel for the $D_{\alpha}$ and right panel for the $D_{\gamma}$. It is interesting to note that even if only the separatrix is used from the equilibrium as input in the algorithm, the shape of the emissivity follows in some way the flux surfaces. The shape of $\epsilon_{\alpha}$ follows the curvature of the flux surface in the inner side and over the dome; $\epsilon_{\gamma}$ instead is concentrated along the separatrix, from the X-point to the divertor target. This is an indirect confirmation that the algorithm works and that the (few) assumptions made are correct: in fact, since deuterium emission depends also on $n_{e}$ and $T_{e}$, it should follow at least partially the flux surfaces, with some corrective effect due to the dependence on neutrals.

\section{NEUTRAL DENSITY EVALUATION}

From the emissivity of the two Balmer lines, it is possible to obtain an estimate of the neutral density in the divertor region. As a matter of fact, the emissivity of the two D lines mainly depends on neutral density $n_{0}$, electron density $n_{e}$, electron temperature $T_{e}$ and ion density $n_{i}$, and can be modeled as:

$$
\begin{aligned}
& \epsilon_{\alpha}=P E C_{\alpha}^{e x}\left(n_{e}, T_{e}\right) n_{0} n_{e}+P E C_{\alpha}^{r e c}\left(n_{e}, T_{e}\right) n_{e} n_{i} \\
& \epsilon_{\gamma}=P E C_{\gamma}^{e x}\left(n_{e}, T_{e}\right) n_{0} n_{e}+P E C_{\gamma}^{r e c}\left(n_{e}, T_{e}\right) n_{e} n_{i}
\end{aligned}
$$

where the first term accounts for the collisional excitation and the second one the recombination. In the paper $n_{i}=n_{e}$ is assumed for solving these equations. The photon emission coefficients (PECs) depend on the electron temperature and density. They are obtained from ADAS database [26].

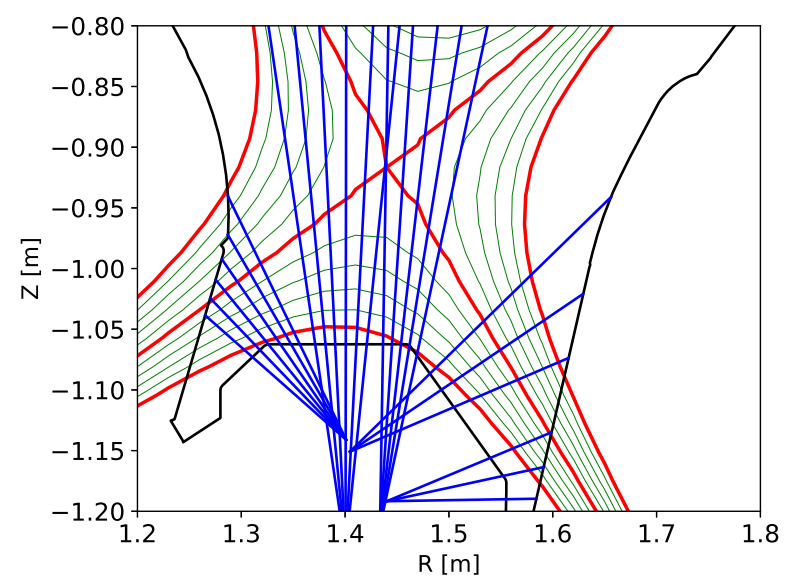

Figure 3: Lines of sight of the Stark diagnostic that look in the divertor region (blue). Vessel and magnetic flux surfaces are also shown. 


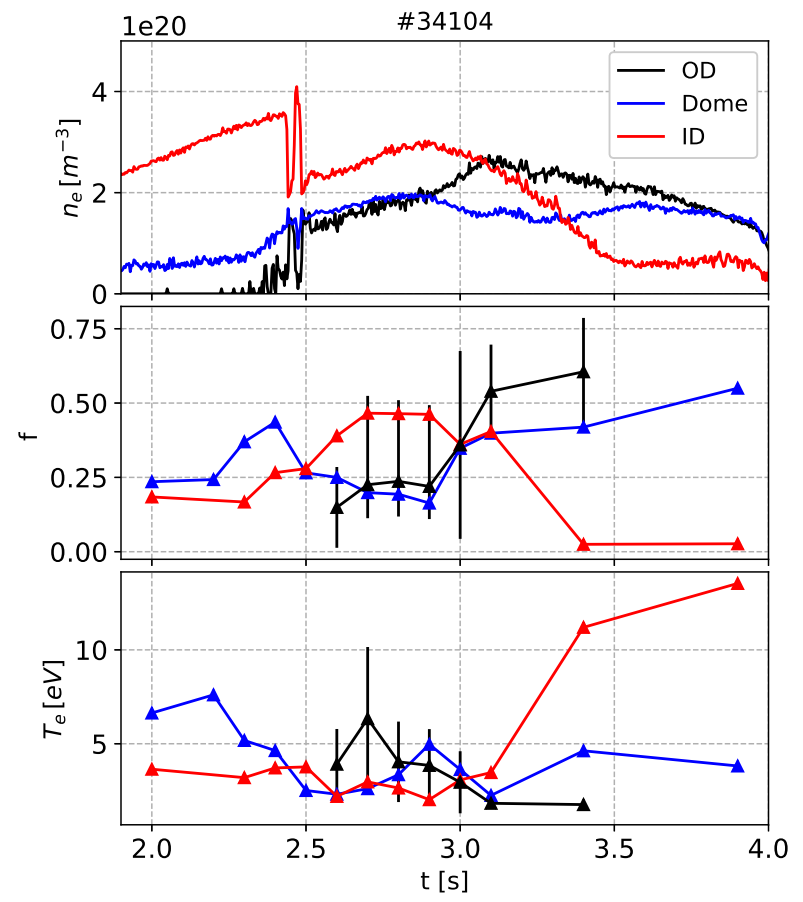

Figure 4: Example of the obtained result. Electron density measured by Stark diagnostic (top), neutral fraction $\left(f=n_{0} / n_{e}\right)$ (middle) and electron temperature (bottom) estimated by inverting equations 3 . The three colours refer to three different Stark lines of sight: black for the one looking in the outer divertor, blue in the dome region, red in the inner divertor. The errorbars in $f$ and $T_{e}$ is the $1 \sigma$ dispersion of the estimates along the LoS. They are shown only for the outer divertor measurement, but they are similar also for the red and blue signals.

Measuring only two Balmer lines, the system is not resolvable, since it has three unknowns: $n_{0}, n_{e}$ and $T_{e}$. However, the electron density in the divertor is measured by the Stark broadening diagnostic [27], with the limitation that it measures line integrated signals, so a real 2D measure of $n_{e}$ is not available. The arrangement of the lines of sight of the Stark diagnostic that observe the divertor region is shown in fig. 3. Following the paper [27], the LoSs that look at the inner and outer divertor are identified with their normalized radius $\rho$ where they intercept the targets; the LoS that look a in the private region over the dome are labeled with their radial $\Delta R$ from the $\mathrm{X}$-point (positive/negative $\Delta R$ means right/left respect the $\mathrm{X}$-point). This diagnostic measures the maximum $n_{e}$ along each set of LoSs. So for each LoS there is a $n_{e}$ measure, and a set of different estimates of $D_{\alpha}$ and $D_{\gamma}$ emissivities, one for each pixel along the Stark LoS. For all the pixels along one LoS, the system of equations 3 is solved via $\chi^{2}$ minimization, obtaining for each pixel the couple $\left(n_{0}, T_{e}\right)$. If the difference between the experimental measurement of $D_{\alpha}$ and $D_{\gamma}$ 
emission and the one obtained using the solution of equation 3 is larger than $20 \%$, this solution is discarded. This can happen because of two reasons: the electron density assigned to a certain pixel is not correct; or the model of the emission described in equation 3 is not valid in those pixels. This means that processes other that excitation and recombination may play a role in populating energetic D levels. In particular, contribution from molecules can be important, especially at low temperature [28-30].

Then, for each line of sight the average neutral density and electron temperature is evaluated, and the results for shot 34104 are reported in figure 4. Time evolution of $n_{e}, T_{e}, f=n_{0} / n_{e}$ for three Stark LoS is reported: black for outer divertor (OD), blue for the dome, red for the inner divertor (ID). This figure highlights also the limit of this analysis, which is the lack of a 2D spatial resolution. In particular, it is possible to obtain only an average value of $n_{0}$ and $T_{e}$ along each Stark line of sight, since for each LoS different neutral emissivity are estimated with the tomographic inversion, but only one $n_{e}$ is measured. The dispersion of the results is highlighted with the errorbars in Fig. 4. For this particular discharge (which will be described and analysed in the next section), neutral density is between 0.2 and $0.7 n_{e}$, and $T_{e}$ is less then $5 \mathrm{eV}$.

In order to verify the electron temperature and the neutral density obtained from $D_{\alpha}$ and $D_{\gamma}$ cameras, these are compared with the ones obtained by different diagnostics. For $T_{e}$ measurements Langmuir probes installed in the divertor target plates are used. Figure 5 compares, as a function of time, the electron temperature measured by the probes at different normalized radii $\rho$ (trian-

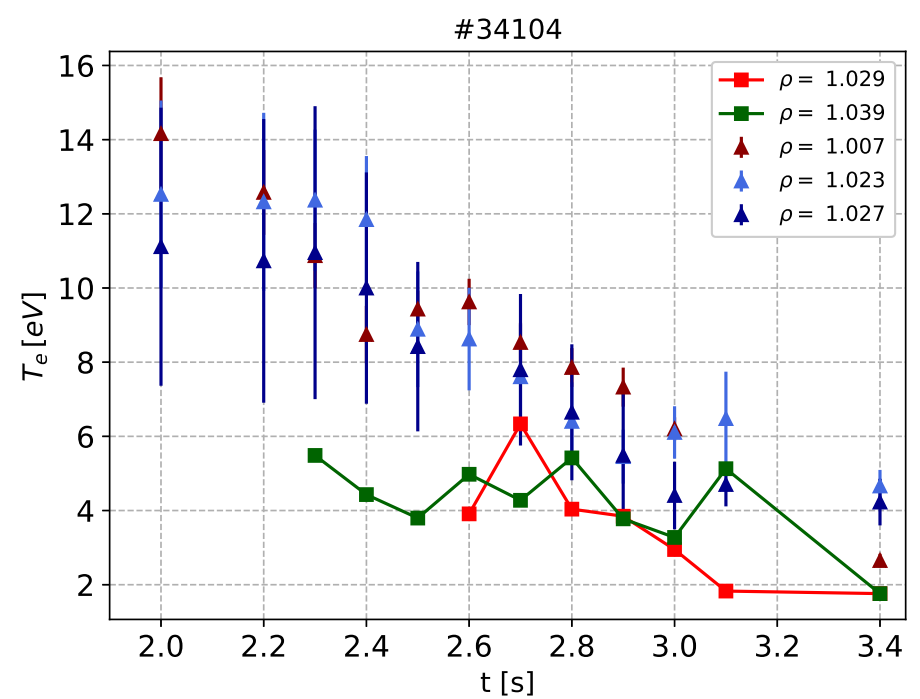

Figure 5: Electron temperature estimated by the Langmuir probes installed at the outer divertor target (triangles) at different $\rho$ (see the legend), and by the spectroscopic method (squares). 

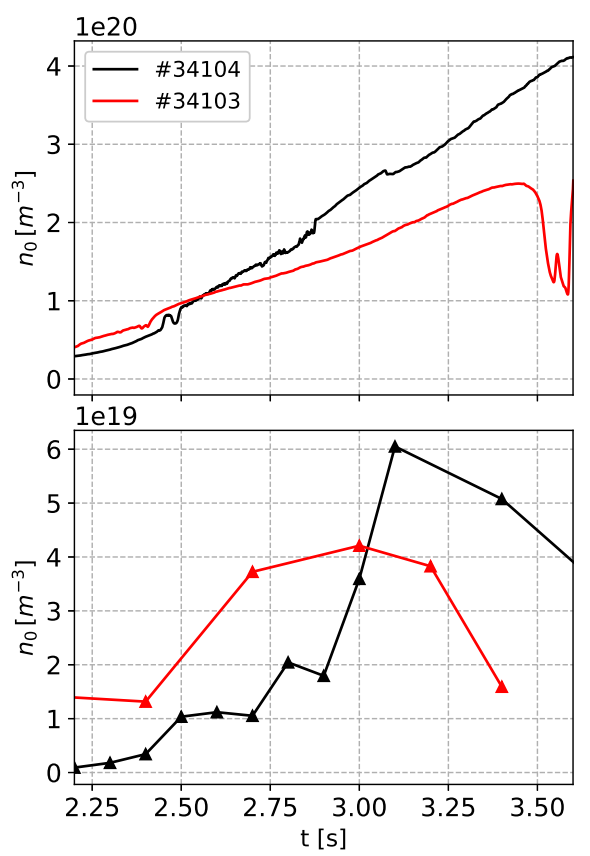

Figure 6: Neutral density behaviour estimated by pressure gauge (top panel) and by spectroscopic method (bottom panel). The gauge measures the molecular pressure, instead the spectroscopic method the atomic ones. In the bottom panel, neutral density is estimated for an outer target LoS. The two colours refer to the two discharges reported in the legend

gles) and the average electron temperature of the pixels near the outer divertor target obtained by the spectroscopic measurements (squares). In this paper in order to identify the position of the probes along the outer divertor target we preferred to use the normalized radius $\rho$ instead of the geometrical length coordinate $s$ along the target. The time behaviour of the two measurements is similar, with $T_{e}$ decreasing in time. The values instead are different, with the probes measuring always a higher temperature. This can be ascribed to the different positions of the measurements: the probes are at the target, instead the Stark diagnostic covers a different region. The electron temperature measured by the spectroscopic method is around $4-7 \mathrm{eV}$ for $t<2.8 \mathrm{~s}$; afterwords, it decreases to around $2 \mathrm{eV}$, where recombination is expected to play a major role with respect to excitation.

The comparison for the neutral density is more difficult, since it is possible to compare it only with the molecular density inferred by the divertor pressure gauges [31] mounted below the divertor plates. In particular, Fig. 6 compares the time behaviour of $n_{0}$ (atomic) estimated by the spectroscopic method in the outer divertor region with the molecular density for two discharges. Both the gauge and the spectroscopic data show that $n_{0}$ increases with time; the two trends are similar, 
which is expected if the dissociation of molecules does not change in time.

\section{EXPERIMENTS WITH SHOULDER FORMATION}

The spectroscopic technique described in the previous paragraph for measuring the distribution of the neutrals in the divertor region is applied to a set of different L-mode discharges of ASDEX Upgrade, characterized by density ramps which can lead to a disruption. The main goal is to shed light on the role of neutrals in the detachment and shoulder formation. As an example, figure 7 compares two discharges at two different plasma currents and with electron density ramp obtained trough deuterium puffing. In both discharges the deuterium puffing from the divertor

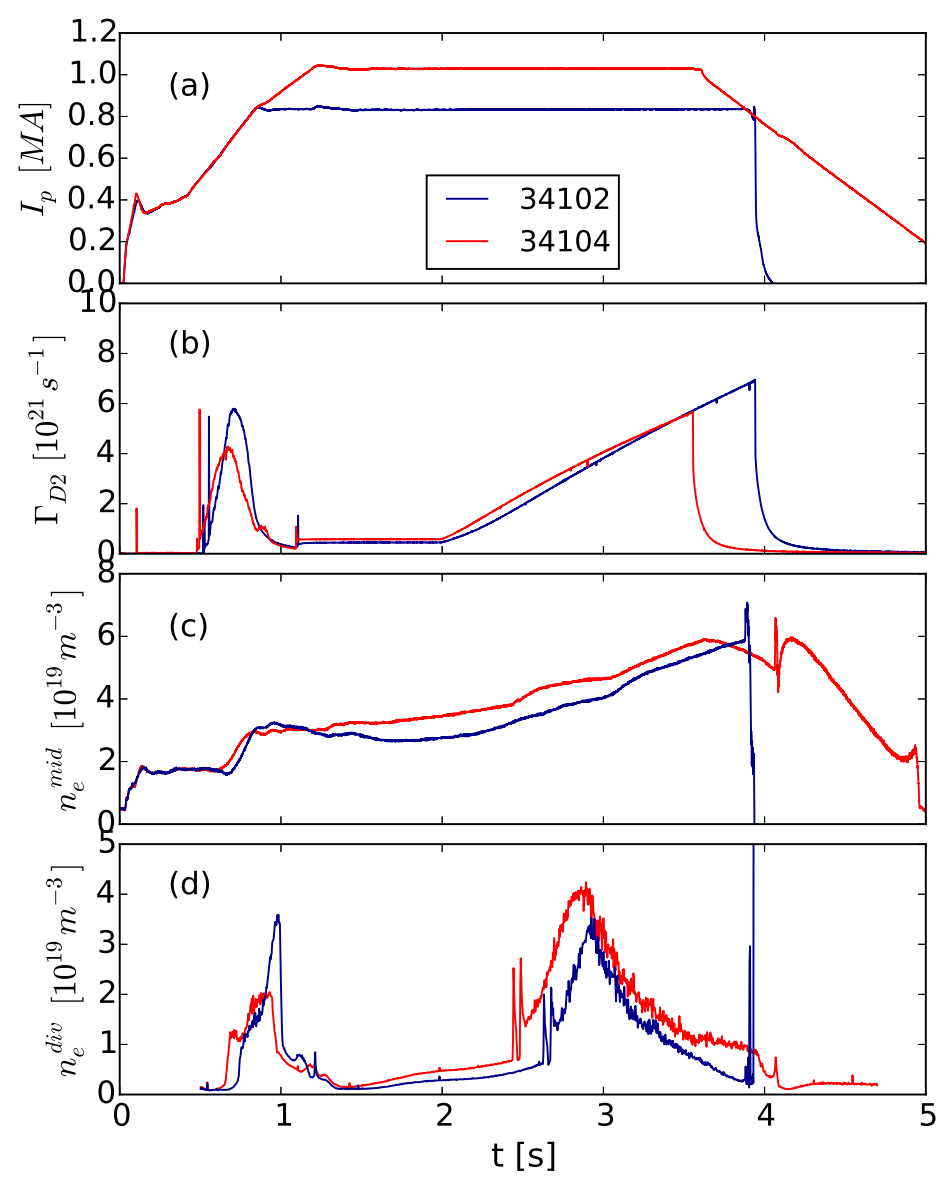

Figure 7: Plasma parameters of two L-mode discharges. Plasma current (a), flux of puffed deuterium (b), midplane electron density measured by a central chord of the interferometer (c) and divertor electron density measured by Langmuir probes installed in the outer divertor target at $\rho \approx 1.03(\mathrm{~d})$. Toroidal field is $3.1 \mathrm{~T}$ for \#34104 and $1.9 \mathrm{~T}$ for \#34102. 


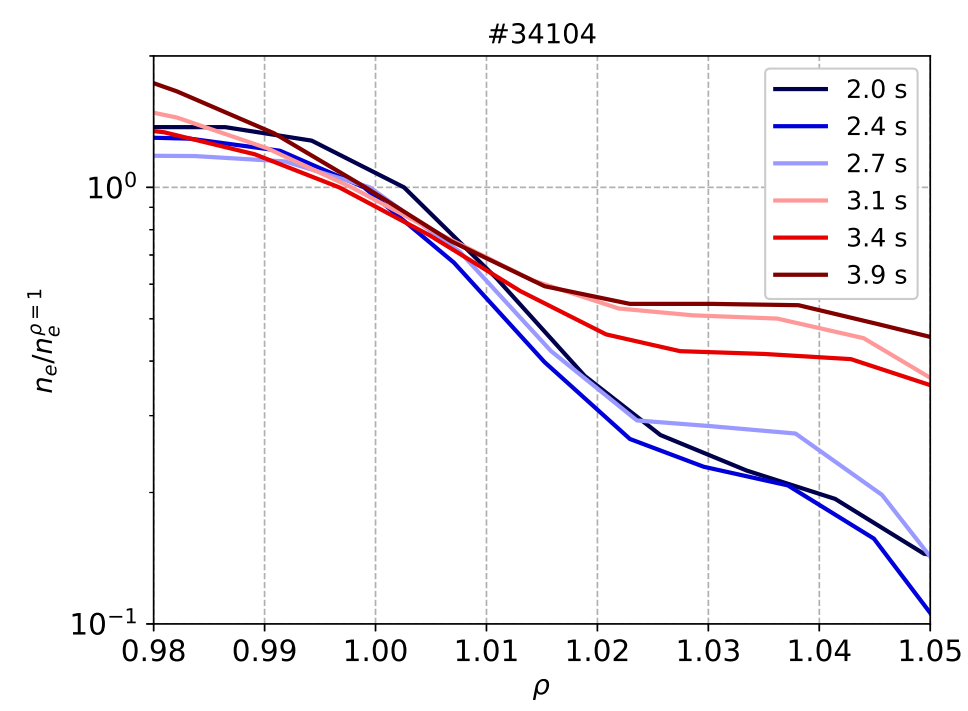

Figure 8: Midplane electron density profile normalized to $n_{e}$ at the separatrix estimated with the Li beam diagnostic. Different colors refer to different time instants as indicated in the legend. In this discharge the roll-over is at $t \approx 2.9 \mathrm{~s}$. Shoulder starts to form after $t=2.7 \mathrm{~s}$; after the roll-over the shoulder in the midplane electron density is well formed, and $n_{e}$ at $\rho>1$ does not change its shape.

starts at $t=2 \mathrm{~s}$, and it causes an increase of the central density up to the density limit (7 (b)). The divertor electron density shows a different behaviour (d): a strong increase is found after the puffing has started, at around $t=2.5 \mathrm{~s}$ for the $1 \mathrm{MA}$ discharge, and later, around $t=2.7 \mathrm{~s}$, for the one at lower current. Then, at about $t=3 \mathrm{~s}$, the central electron density continues to increase, while the divertor density collapses. This is the density roll-over, a clear evidence of detachment. In the two discharges the roll-over happens at two slightly different central electron densities: shot \#34102 with a lower plasma current, detaches at lower midplane density. The radial profiles of $n_{e}$ at the outboard midplane measured with the Li-beam diagnostic [33] evolve as reported in figure 8. This figure highlights the development of the shoulder in the midplane electron density: after the deuterium puffing, at $t \approx 2.8 \mathrm{~s}$ (see the red lines of figure 7 ), outside the separatrix $n_{e}$ increases with respect to its value at the separatrix, and the edge electron profile flattens.

With the density roll-over and the detachment, the electron density behaviour at the outboard midplane $\left(n_{e}^{\text {mid }}\right)$ and in the divertor region $\left(n_{e}^{d i v}\right)$ are quite different: $n_{e}^{\text {mid }}$ increases with time until the end of the discharge, while $n_{e}^{d i v}$ increases only during the first part of the puffing and then collapses. This behaviour, for the same discharge, is shown in Fig. 9. Here the electron density at the outer divertor target measured with two different diagnostics (a Langmuir probe installed in the divertor plate, with blue-green colors, and the Stark broadening diagnostic in violet-yellow 


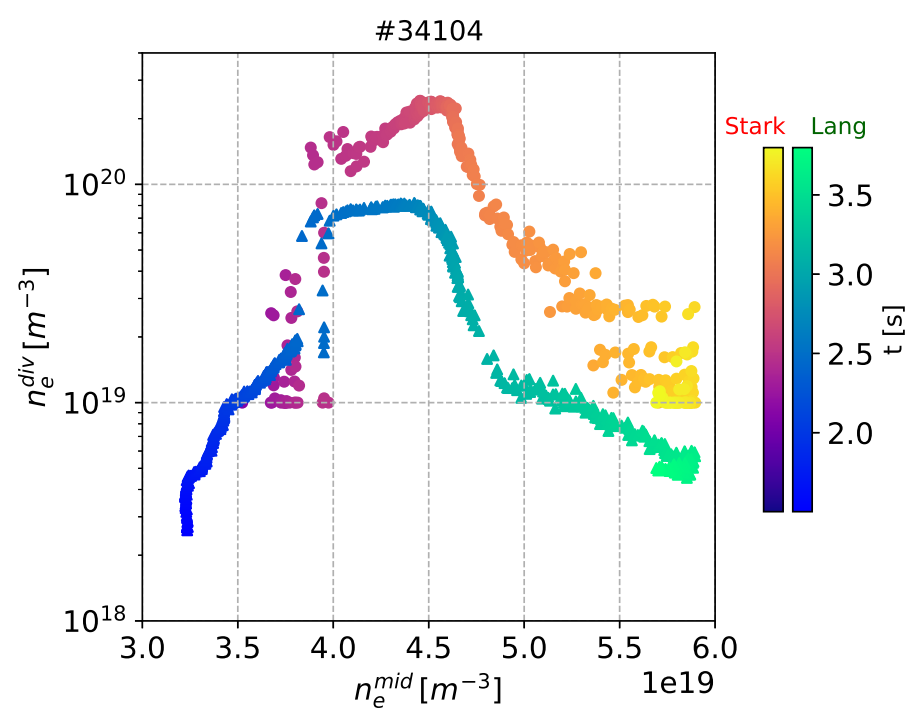

Figure 9: Electron density at/near the outer divertor target as a function of the central electron density at the midplane. In the divertor, $n_{e}$ is measured with a Langmuir probe (blue-green colors) and with the Stark broadening diagnostic (violet-yellow colors); at the midplane, it is measured with the interferometer. Color codes refer to the time of the discharge. Roll-over starts at $t \approx 2.9 \mathrm{~s}$, when $n_{e}^{\text {mid }} \approx 4.5 \cdot 10^{19} \mathrm{~m}^{-3}$.

colors) is plotted as a function of the central midplane density measured with the interferometer. With the deuterium puffing the midplane density continuously increases during the whole duration of the discharge; the divertor density increases up to $n_{e}^{m i d} \approx 4 \cdot 10^{19} \mathrm{~m}^{-3}$; then its variation slowsdown, and the midplane shoulder begins to form. When the midplane density increases further $\left(n_{e}>4.5 \cdot 10^{19} \mathrm{~m}^{-3}\right)$, the divertor density collapses: this last part corresponds to the roll-over phase and the detachment. The electron densities measured by the two divertor diagnostics have a similar time-behaviour, however the Stark estimate is systematically larger than the Langmuir one. This difference may be ascribed to the slightly different regions measured by them: Langmuir probes are installed in the divertor plates; the Stark broadening diagnostic measures along lines of sight across the divertor region, and probably its density measurement can be located in a volume where the LoS intersects the separatrix.

During the evolution of the $n_{e}$ profile the divertor emission also changes, as reported in figure 10 where $\epsilon_{\alpha}$ and $\epsilon_{\gamma}$ are shown in the first and second row, respectively. During the D puffing but before the strong increase of the divertor density $(t<2.5 s)$, the emissivity profiles of $D_{\alpha}$ and $D_{\gamma}$ are very similar: the strongest emission is localized on the high field side, in the inner divertor region, while it is very low in the outer divertor region. Then at $t=2.7 \mathrm{~s}$, when the divertor $n_{e}$ measured by Langmuir probes and the Stark broadening diagnostic increases (Fig. 7) and when the shoulder 


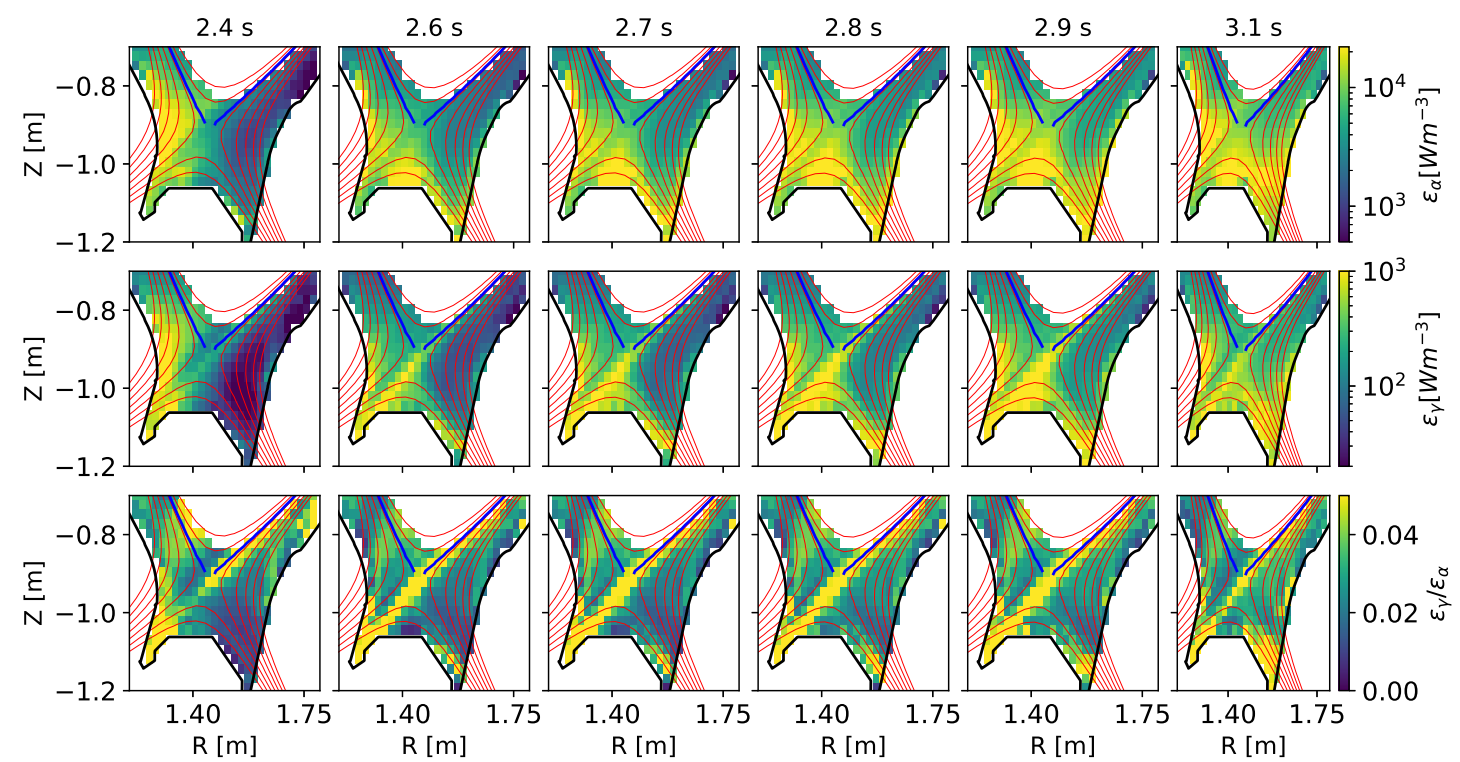

Figure 10: Time evolution of $\epsilon_{\alpha}$ (top row) and $\epsilon_{\gamma}$ (central row) emissivities, and the the ratio $\epsilon_{\gamma} / \epsilon_{\alpha}$ (bottom row) for shot \#34104. Each row has the same colorbar reported on the right side. Time instants are indicated in the top row. In each contour the flux surfaces are indicated in red and the separatrix with the thick blue line. Deuterium puffing begins at $\mathrm{t}=2 \mathrm{~s}$, divertor density strongly increases around $\mathrm{t}=2.6 \mathrm{~s}$, and the roll over is at $\mathrm{t}=2.9 \mathrm{~s}$ (see figure 7 ).

starts to form, the emissivity moves toward the low field side region near the outer divertor leg and the dome, and their values increase. For $t>2.9 \mathrm{~s}$, with the density roll-over and a well established shoulder, the neutral emission is spread in the whole divertor region, reaching also the separatrix, with a further increase of the values. This behaviour reflects in first approximation the behaviour of the electron density, as described in [34]. In the bottom panels the ratio of the two D emissivities $\epsilon_{\gamma} / \epsilon_{\alpha}$ is shown, and this ratio can be used as a raw indication of the role of recombination process in the neutral deuterium emission. If only excitation is taken into account in equations 3 , the ratio $\epsilon_{\gamma} / \epsilon_{\alpha}$ is always lower than 0.03 for $2<T_{e}<500 \mathrm{eV}$ and $n_{e}>5 \cdot 10^{18} \mathrm{~m}^{-3}[12,16]$. In the attached phase $(t=2.4 s)$ excitation dominates in most of the divertor region; then during the high recycling phase $(t \geq 2.6 s)$ this ratio is large along the separatrix, from the $\mathrm{X}$-point to the inner divertor target, suggesting that recombination is starting to play a role. Finally in the last time instant, in the detached phase, recombination is important also in the outer target, with a large value of the ratio $\epsilon_{\gamma} / \epsilon_{\alpha}$.

The same information can be found also in figure 11. Here the time behaviour of the ratio $\epsilon_{\gamma} / \epsilon_{\alpha}$ in the outer divertor region is compared with the electron density measured at the outer target 


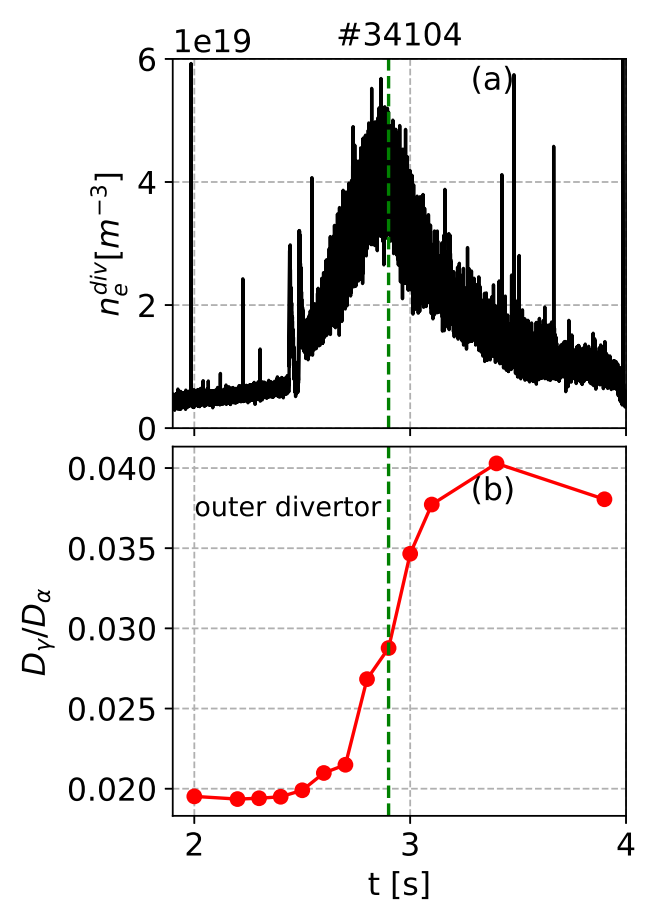

Figure 11: Electron density at the outer divertor target measured by a Langmuir probe (a), and ratio $\epsilon_{\gamma} / \epsilon_{\alpha}$ in the outer divertor region (b). The vertical green dashed line is the time instant of the density roll-over and detachment.

by a Langmuir probe. After the detachment, in the outer divertor region this ratio reaches values up to 0.04 , or even larger, which cannot be explained by excitation process only. However, the recombination may play a role also before detachment, even if smaller than the excitation. In fact, $n_{e}$ at the outer divertor shown in figure 11 increases continuously for $t>2 \mathrm{~s}$; if the Balmer lines were due to excitation only, the ratio $D_{\gamma} / D_{\alpha}$ would decrease with increasing $n_{e}$. Instead the figure shows that the ratio is constant before $2.6 \mathrm{~s}$, then it increases together with $n_{e}$ up to the roll-over. This is a clear indication of a relation between recombination and shoulder formation.

A quasi-2D picture of the behaviour of $n_{e}, T_{e}, n_{0}$ in the divertor for discharge 34104 , during attached, high recycling and detached phases is summarized in figure 12. During the first part of the puffing (attached phase, $2<t<2.7 s$ ) electron density is larger in the inner target; in the high recycling regime, $n_{e}$ starts to increase also in the private region over the dome and in the outer target; finally, with the roll-over it becomes very low in the inner target, in the private region its maximum moves toward positive $\Delta R$ (i.e. toward the low filed side) and upward along the outer divertor target. Neutral density is maximum in the inner target during the attached phase; then in the high recycling regime with the shoulder formation it increases in the high field side and in the private region over the dome, and it starts to be measurable also at the outer divertor target. 

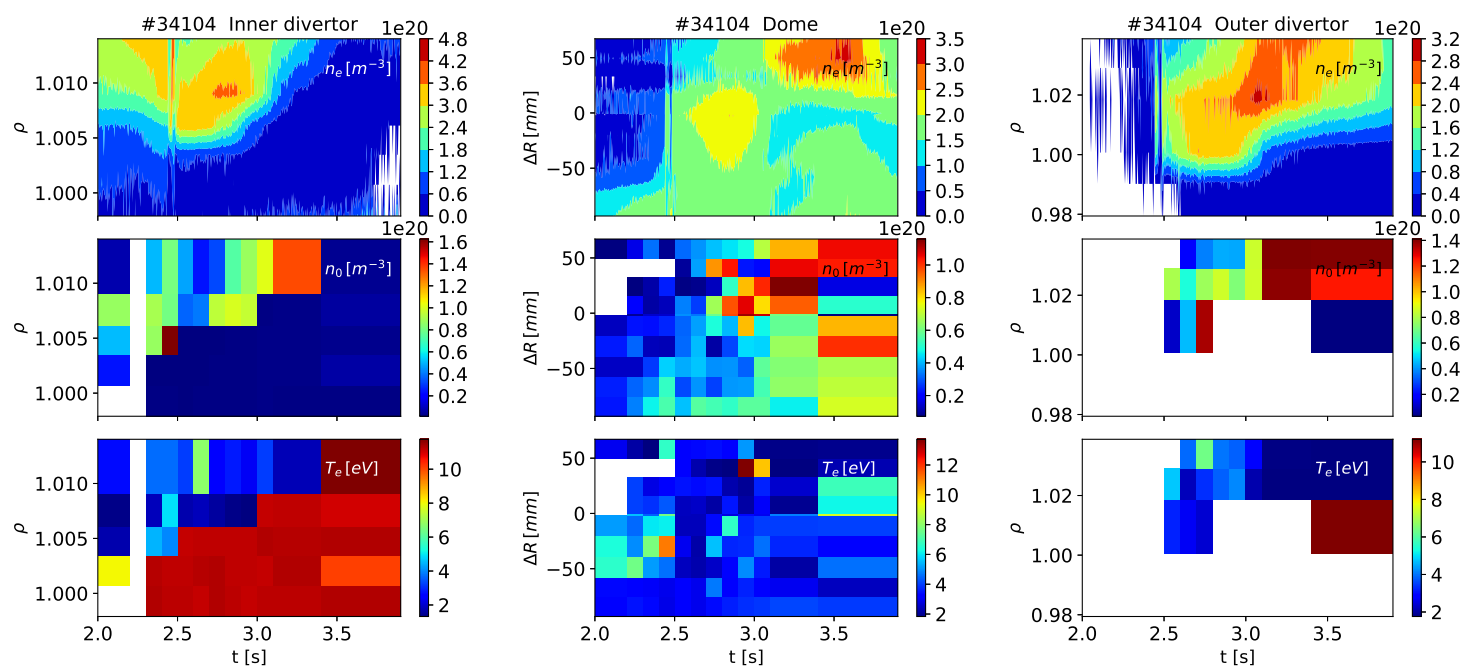

Figure 12: Time evolution of $n_{e}, n_{0}$ and $T_{e}$ in the divertor region. Left panels refer to inner divertor measurement; central panels refer to the dome region; right panels to outer divertor. Top: electron density measured by Stark diagnostic; center: neutral density estimated by the spectroscopic technique; bottom: electron temperature estimated by spectroscopic technique. The Stark LoS in the inner and outer divertor are labeled according the $\rho$ value of their intersections with the divertor target; for the dome, they are labeled according to the distance respect to the X-point $\Delta R$.

Then after the density roll-over $(t=3.1 \mathrm{~s}) n_{0}$ increases further, with large values in the whole private region over the dome and at the outer target. Electron temperature estimated with this spectroscopic technique shows a specular behaviour. With shoulder formation and the increase of $n_{e}$ and $n_{0}$, it decreases. For $t>2.5 \mathrm{~s}$ it is very low $(\approx 2 \div 4 \mathrm{eV})$ in the private region and outer target. It is large, around $10 \mathrm{eV}$ only at the inner target, where $n_{e}, n_{0}$ decrease.

Figure 13 tries to summarize the behaviour of electron and neutral density (panels a and b) in different regions of the divertor during the discharge, comparing them with the midplane SOL $n_{e}$ radial profile: panel (c) shows the characteristic radial decay length of the midplane SOL electron profile, estimated as $\lambda_{n}=-n_{e} / \nabla n_{e}$ in the region $1.01<\rho<1.07$. With deuterium puffing, up to $t=2.5 \mathrm{~s}$, the divertor is in a low recycling regime; the electron density profile in the SOL midplane is steep $\left(\lambda_{n} \approx 20 \mathrm{~mm}\right)$; in the divertor $n_{e}<10^{20} \mathrm{~m}^{-3}$ and neutral density is about $10^{19} \mathrm{~m}^{-3}$ in the private region, and not measurable in the outer target. Continuing the puffing, the discharge moves to high recycling regime, at $t \approx 2.6 \mathrm{~s}$. In this regime, the shoulder begins to form as $\lambda_{n}$ increases; panel (a) shows that in the outer divertor target at $\rho>1$ electron density continuously rises, as in the private region. Density of neutrals also raises: neutral fraction assumes values between 0.2 and 0.4 , and it is larger in the private region (red) respect to the outer target divertor SOL. Then 

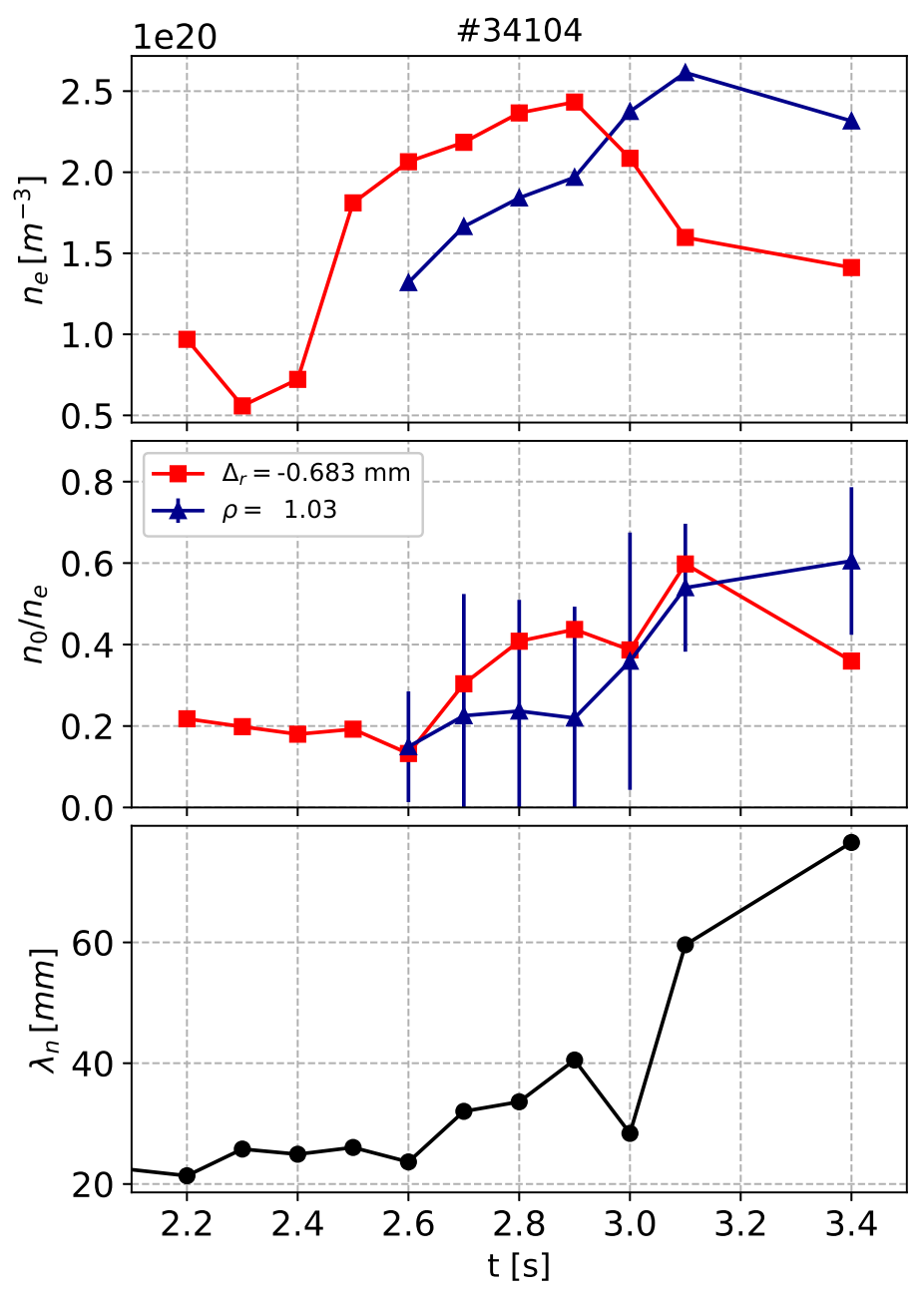

Figure 13: Time evolution of electron density (a), neutral density fraction (b) and e-folding length of the midplane SOL density profile (c). Red square points refer to dome region, blue triangles to outer divertor.

in the last part of the discharge, the roll-over and detachment, the SOL profile continues to flatten $\left(\lambda_{n}>40 \mathrm{~mm}\right) ; n_{e}$ near the X-point has reached its maximum and decreases, assuming values lower than at the outer target (panel (a)); panel (b) shows that $n_{0}$ is about 0.6 times $n_{e}$ both in the private region and in the SOL outer target.

The neutral behaviour described in figures 11 and 13 is in qualitative agreement with the simulations of EMC3-EIRENE reported in [11]: at higher electron density, when the shoulder is formed, in the divertor the neutral density increases in the entire private flux region, from the target up to the separatrix and the X-point. Moreover figures 12 and 13 shows that moving from low to high core $n_{e}$ the distribution of $n_{0}$ and $T_{e}$ in the divertor becomes more symmetric; after the roll over, the whole private region over the dome and the outer divertor have similar $T_{e}$ and $n_{0}$. This 
symmetry at higher density is also observed in SOLPS simulations with drifts in [35].

\section{DISCUSSION AND CONCLUSIONS}

The exploitation of two visible cameras looking at the lower divertor region of ASDEX Upgrade allows to obtain 2D maps of neutral emissivity and an estimate of the neutral density. With the developed tomographic algorithm, the $2 \mathrm{D}$ maps of $\epsilon_{\alpha}$ and $\epsilon_{\gamma}$ in the divertor region are obtained from line integrated measurements; from this the density of the neutral atomic deuterium can be obtained via equations 3. In particular the behaviour of neutrals for attached and detached divertor plasmas has been studied, trying to understand their role in the formation of the density shoulder in high density and detached plasmas.

Before summarizing the obtained results, it is important to remind the assumptions that have been made. In the tomographic algorithm, the reflection on the tungsten divertor tiles is not considered. Adding reflection in the tomographic model is very complicated since the reflectivity depends on the angle between the material and the line of sights, and on the surface of the tiles itself, that can change during operation, especially with strong plasma-wall interaction expected in the divertor [36, 37]. In [38] the results of a tomographic model with and without reflections in ASDEX Upgrade are compared, and it is concluded that in the divertor region the differences in the reconstructed emissivity are not significant. Similar conclusions are reported also in [39] for JET. Moreover, in [40, 41], simulations done for ITER show that in the divertor region, where the expected plasma emission is large, reflections are not so important. Another assumption is not having considered the molecules in equation 3. Their contribution can be important when calculating the emissivity of Balmer lines especially in a detached divertor, when $T_{e}$ is very low $(0.5-5 \mathrm{eV})$, as discussed in the paper. Moreover $n_{0}$ and $T_{e}$ estimated with the spectroscopic technique are an average value along the Stark diagnostic lines of sight, with no localization along the lines themselves.

With this in mind, the analyses described in the paper give some indications of the role of divertor neutral density in midplane density shoulder formation and detachment. When the midplane $n_{e}$ increases, shape and values of neutral Balmer emission in the divertor change: the emission is larger in the inner divertor at low density, and when $n_{e}$ increases, neutral emission moves towards the dome and the outer divertor, increasing also its value. The neutral density increases too, being always higher in the inner divertor than in the outer one, but at density roll-over. After the roll-over, when the shoulder in midplane SOL $n_{e}$ profile is well formed, neutral fraction increases 
everywhere in the divertor, assuming similar values in the private region and at the outer target. In the inner target $n_{0}$ is smaller, following the behaviour of the electron density.

The possible next steps for a better comprehension of the divertor neutrals time evolution and their role on the upstream electron density profile modification following the work described in this paper, are of two types: modeling and experimental measurements improvements. Detailed SOLPS simulations of the discharges here analysed are foreseen, to be compared with the experimental results; moreover, a better divertor measurement of $n_{e}$ and $T_{e}$ profiles would be available with the new divertor Thomson scattering diagnostic, which is going to be installed. It will provide a local measurement of electron density and temperature, and together with the $2 \mathrm{D} D_{\alpha}$ and $D_{\gamma}$ emissivities will help to improve the estimate of the neutral density.

\section{ACKNOWLEDGMENTS}

This project has received funding from the European Unions Horizon 2020 research and innovation program under grant agreement number 633053. The views and opinions expressed herein do not necessarily reflect those of the European Commission. 
[1] G.Federici et al., Nucl.Fusion, 41, 1967, (2001)

[2] K.Borrass, G.Janeschitz, Nucl.Fusion, 34, 1203, (1994)

[3] C.S.Pitcher and P.C.Stangeby, Plasma Phys.Control.Fusion, 39, 779, (1997)

[4] B.Lipschultz, B.LaBombard, J.L.Terry, C.Boswell and I.H.Hutchinson, Fusion Science and Technology, $\mathbf{5 1}, 369,(2007)$

[5] A.W.Leonard, Plasma Phys.Control.Fusion, 60, 044001, (2018)

[6] P.C.Stangeby, Nucl.Fusion, 33, 1695, (1993)

[7] A.Wynn et al., Nucl.Fusion, 58, 056001, (2018)

[8] D.Carralero et al., Phys. Rev. Lett., 115, 215002, (2015)

[9] N.Vianello et al. Nucl.Fusion, 57, 116014, (2017)

[10] B.Lipschultz, D.Whyte and B.LaBombard, Phys. Plasmas 47, 1559, (2005)

[11] D.Carralero et al., Nucl.Fusion, 57, 056044, (2017)

[12] T.Fujimoto, S.Miyachi and K.Sawada, Nucl.Fusion, 28, 1255, (1988)

[13] J.A.Goetz et al., Phys. Plasmas 3, 1908, (1996)

[14] C.Kurz et al., Plasma Phys.Control.Fusion, 39, 963, (1997)

[15] C.J.Boswell et al., Journal of Nuclear Materials, 290-293, 556, (2001)

[16] A.Huber et al., Journal of Nuclear Materials, 438, S139, (2013)

[17] B.A.Lomanowski, A.G.Meigs, R.M.Sharples, M.Stamp, C.Guillemaut and JET Contributors, Nucl. Fusion, 55, 123028, (2015)

[18] K.Verhaegh et al., Nuclear Materials and Energy, 12, 1112, (2017)

[19] M.Carr et al., Rev.Sci.Instrum, 89, 083506, (2018)

[20] F.Natterer, The Mathematics of Computerized Tomography, Teubner, Stuttgart, 1986

[21] G.T.Herman, Real Time Imaging, 1, 3, (1995)

[22] F.Natterer, Acta Numerica, 107, (1999)

[23] P.C.Sabatier, J.Math.Phys., 41, 4082, (2000)

[24] A.H. Andersen and A.C.Kak, Ultrasonic Imaging, 6, 81, (1984)

[25] M.Agostini et al., Phys. Rev. ST Accel. Beams, 14, 102801, (2011)

[26] http://www.adas.ac.uk

[27] S.Potzel, R.Dux, H.W.Müller, A.Scarabosio, M.Wischmeier and ASDEX Upgrade team, Plasma Phys.Control.Fusion, 56, 025010, (2014)

[28] J.L.Terry et al., Journal of Nuclear Materials, 266-269, 30, (1999)

[29] K.Behringer and U.Fantz, New Journal of Physics, 2, 23, (2000)

[30] U.Fantz et al., Journal of Nuclear Materials, 290-293, 367, (2001)

[31] A.Scarabosio and G.Haas, AIP Varenna Conference Proceedings, 998, 238, (2008)

[32] N.Vianello et al., Scrape off layer transport and filamentary dynamics in high density tokamak regimes, 
$27^{\text {th }}$ IAEA-FEC 2018, EX/P8-13

[33] M.Reich, E.Wolfrum, J.Schweinzer, H.Ehmler, L.D.Horton, J.Neuhauser and ASDEX Upgrade Team, Plasma Phys.Control.Fusion, 46, 797808, (2004)

[34] S.Potzel et al., Nucl.Fusion, 54, 013001, (2014)

[35] L.Aho-Mantila et al., Nucl.Fusion, 59, 035003, (2017)

[36] E.M.Hollmann et al., Rev.Sci.Instrum, 74, 3984, (2003)

[37] E.M.Hollmann et al., Contrib.Plasma Phys., 44, 301, (2004)

[38] J.Harhausen et al., Plasma Phys.Control.Fusion, 53, 025002, (2011)

[39] M.Carr et al., Physically principled refection models applied to filtered camera imaging inversions in metal walled fusion machines, sibmitted to Rev.Sci.Instrum.

[40] S.Kajita et al., Journal of Nuclear Materials, 936-939, 463, (2015)

[41] S.Kajita et al., Nucl.Fusion, 57, 119061, (2017) 